\title{
Fabricación de componentes constructivos con la fracción plástica de residuos provenientes del reciclado de RAEE
}

\section{Manufacture of construction components with plastic fraction of WEEE recycling waste}

Presentación: 11/10/2019

\section{Doctorando:}

\section{Melina Gabriela Gómez}

Instituto de Física Aplicada (INFAP)

dimelinag@gmail.com

\section{Director/es:}

\section{Dr. Jerónimo Kreiker - Director \\ Dr. Lucas Peisino - Codirector \\ Dra. Belén Raggiotti - Codirectora}

\section{Resumen}

Los residuos de aparatos eléctricos y electrónicos (RAEE), han crecido notablemente en cantidad en los últimos años y se espera que esta tendencia continúe. La fracción plástica del desecho (FPR), constituye aproximadamente el 30\% del total en peso, y no ha podido ser insertada en el circuito del reciclado, debido a la heterogeneidad de plásticos constitutivos y a la presencia de contaminantes, principalmente retardantes de llama y metales pesados, ambos tóxicos para la salud humana.

Existen numerosos estudios sobre el agregado de plásticos a morteros, actuando como material de carga o fibras de refuerzo, logrando materiales con aptitud para este uso. El estudio de estos antecedentes llevó a considerar el uso de la FPR como agregado en morteros, en reemplazo totaly parcial del agregado tradicional.

Se desarrolló un agregado sintético (AS) mediante la estrategia del core-shell, en el cual el núcleo de plástico es recubierto con sucesivas capas de cemento y se estudió su desempeño como componente constructivo mediante ensayos de resistencia mecánica y determinación de propiedades físicas. El AS puede ser utilizado en morteros en reemplazo totaly parcial de agregado fino, sin disminuir significativamente la resistencia mecánica. A pesar de los buenos resultados que se obtuvieron, con el paso del tiempo aparecen exudados amarillos en la superficie resultado de la desestabilización de los retardantes de llama en el medio básico del cemento, lo cual lo hace potencialmente peligroso para el uso en construcciones civiles. Para revertir este efecto se agregó al proceso carbón activado (CA) como aditivo estabilizante. El análisis químico mostró una muy buena estabilización de los contaminantes cuando se usa $\mathrm{CA}$ al $3 \%$ en peso. Para evaluar el efecto del CA con el fraguado de cemento, este trabajo presenta los resultados del uso de diferentes tipos y dosificaciones de CA.

Palabras claves: agregado sintético, plástico de RAEE, mortero sustentable. 


\section{Abstract}

Waste electrical and electronic equipment (WEEE), have grown significantly in quantity in recent years and this trend is expected to continue. The plastic waste fraction (RPF) constitutes approximately $30 \%$ of the total weight, and has not been able to be inserted into the recycling circuit, due to the heterogeneity of constitutive plastics and the presence of contaminants, mainly flame retardants and metals heavy, both toxic to human health.

There are numerous studies on the addition of plastics to mortars, acting as filler material or reinforcing fibers, achieving materials with aptitude for this use. The study of these antecedents led to consider the use of RPF as an aggregate in mortars, in total and partial replacement of the traditional aggregate.

A synthetic aggregate (AS) was developed through the core-shell strategy, in which the plastic core is coated with successive layers of cement and its performance as a construction component was studied by means of mechanical resistance tests and determination of physical properties. AS can be used in mortars in total and partial replacement of fine aggregate, without significantly decreasing mechanical strength. Despite the good results obtained over time, yellow exudates appear on the surface, as a result of the destabilization of flame retardants in the basic medium of cement, which makes it potentially dangerous for use in civil constructions. To reverse this effect, activated carbon (CA) was added to the process as a stabilizing additive. The chemical analysis showed a very good stabilization of the contaminants when using 3\% CA by weight. To evaluate the effect of CA with cement setting, this work presents the results of the use of different types and dosages ofCA.

Keywords: synthetic aggregate, WEEE plastic, sustainable mortar

\section{Introducción}

Los plásticos no pueden degradarse de forma natural como los residuos orgánicos, quedan en la naturaleza por cientos de años alcanzando a nivel mundial 150.000.000 toneladas anuales[1]. El caso de los residuos de aparatos eléctricos y electrónicos (RAEE), es uno de los más preocupantes debido al incremento que ha sufrido en las últimas dos décadas [2] y porque posee características comunes al resto de los plásticos, como su baja bio-degradabilidad que hace que se acumule en los basurales obstruyendo los procesos naturales del agua y la tierra y características únicas, como la presencia de metales pesados como el cadmio $(\mathrm{Cd})$ que se emplea como pigmento, estabilizador o catalizador durante el proceso de polimerización de, por ejem plo el ABS [3]-[4] y otros como el Al, $\mathrm{Ca}, \mathrm{Cu}, \mathrm{Fe}, \mathrm{Mg}$, $\mathrm{Ni}, \mathrm{Pb}$ y $\mathrm{Zn}$ en distintas concentraciones [5] y de sustancias químicas tóxicas persistentes que contaminan el medio ambiente y afectan a la salud de las personas [6].La presencia de estos contaminantes en los artefactos eléctricos y electrónicos (AEE) los tornan más contaminantes para el ambiente que cualquier otro residuo sólido. Al ser dispuestos en un basural sin tratamiento previo, los metales pesados y otros compuestos que suelen contener, migran mediante procesos de lixiviación hacia las napas de agua y al ambiente [7]. Resultan peligrosos también para los trabajadores de las plantas de gestión de RAEE, que están expuestos al manejo de ciertas sustancias o desechos que pueden ser riesgosos para su salud y el entorno ambiental.

La fracción plástica de los RAEE forma, en promedio un 34\% del AEE, siendo los componentes electrónicos y los metales los que le siguen en porcentaje [4]. Otra particularidad de esta fracción plástica es su composición heterogénea; está formada por mezclas de: ABS/PC en un 5\%; PMMA: 3\%; PC: 3\%; PA: 1\%; ABS: 29\%; HIPS: 26\%; PP: 22\%; y otros polímeros, $11 \%$. Mientras que los aditivos y cargas que contiene son: fósforo, cloro, antimonio, bromo, cromo, cadmio, plomo, óxido de magnesio, alúmina, silicato, carbonato de calcio y titanio [8].Esta característica hace que su reciclado sea aún más complejo debido a que, para la re-extrusión se requiere un sólo tipo de plástico en la muestra [9] al mismo tiempo que los procesos de separación requerirían un costo extra correspondiente a la 
incorporación de pasos en la cadena de producción, como la selección manual, disolución selectiva, flotación, fluorescencia de rayos X, espectroscopia láser, entre otros [9].

Por otro lado, el plástico de los RAEE (FP RAEE), también por estar en contacto con circuitos electrónicos, debe cumplir con ciertas normas de seguridad establecidas para evitar la propagación de incendios, para esto se utilizan sustancias químicas que se agregan o reaccionan con materiales combustibles para aumentar su resistencia al fuego: los retardantes de llama bromados (BFR's), como clase química incluyen éteres difenílicos aromáticos, cíclicos alifáticos, derivados fenólicos alifáticos, derivados de anhídrido ftálico y otros[10]. Existen actualmente 75 tipos de BFRs disponibles en el mercado, siendo los más comunes y prioritarios los polibromodifeniléteres incluyendo mezclas comerciales de PentaBDE, OctaBDE y DecaBDE, el tetrabromobisfenol A (TBBPA) y el hexabromociclododecano (HBCD) [11], [12] y se aplican en gran cantidad de materiales que requieran de resistencia al fuego, pero es en los aparatos eléctricos y electrónicos donde junto al óxido de antimonio (Sb2O3) como sinérgico [11]en el HIPS y ABS, que se presentan con mayor porcentaje (56\%), le siguen la construcción, los textiles y el transporte. El TBBA es el BFR que usa en la mayoría de los RAEE [9]. Es un polvo cristalino blanco (o incoloro), que contiene un $50 \%$ de bromo[12].

Si bien los retardantes de llama son una solución a la propagación del fuego, pueden salvar vidas y prevenir daños materiales, también son objeto de una creciente preocupación sobre sus impactos medioambientales y efectos sobre la salud ya que no son adecuadamente tratados en la actualidad por el sector informal generando riesgos tanto para los trabajadores como para los consumidores [13]. Algunos de ellos ya han sido probados como perjudiciales para el medio ambiente o los seres humanos, y se ha cesado su producción [10].

Es necesario entonces, llevar a cabo un proceso de reciclaje que considere la existencia de componentes metálicos, aditivos RAEE contiene.

En esta tesis se propone: valorizar el plástico de RAEE reinsertándolo en un ciclo productivo y a su vez, neutralizando la exposición de sus componentes metálicos y contaminantes orgánicos. Se aplicarán estos métodos con el objetivo de generar materiales con recursos recuperados y combinados para ser empleados en componentes constructivos o en construcción de manera directa. El reciclado de desechos y plásticos reciclados en la construcción es una buena alternativa para reducir el desecho plástico. Con este método los plásticos se re aprovechan sin perder calidad y se sustituyen por el uso de los materiales vírgenes en la construcción [15]. Mediante la técnica de core-shell (corazóncoraza) el plástico se cubre con capas sucesivas de cemento de albañilería y carbón activado de origen vegetal. El cemento actúa como estabilizante de metales mientras que el carbón activado como estabilizante de BFR's.

\section{Resultados}

Se desarrolló un árido sintético (AS) que consiste en partículas de plástico de RAEE trituradas y recubiertas con aditivos para ser empleadas en la industria de la construcción como reemplazo total o parcial del agregado fino y grueso natural. Se obtuvo plástico triturado proveniente del RAEE de varios proveedores locales, quienes lo entregan triturado y mezclado en tamaños de partículas de entre 3-8 $\mathrm{mm}$. Las partículas se pesan y se introducen en la máquina mezcladora vertical sin paletas. El cemento y el carbón activado se dosifican y se mezclan previamente. Luego, se incorporan a la mezcladora, donde ya están las partículas y se va agregando el agua hasta completar una relación agua/cemento 0,5. Las partículas se dejan secar 24 horas. Luego de la aplicación de cada capa se realiza un desterronado y tamizado. El AS se puede utilizar a partir de los 7 días, aunque se recomienda su uso recién después de 28 días, lapso considerado óptimo para el fraguado del cemento.

Se realizaron diferentes tipos de muestras con distintas cantidades de estabilizante y aglutinante, algunas con presencia de carbón activado y otras sin él, con el objetivo de evaluar el comportamiento mecánico y las características de las distintas combinaciones. Otras muestras consistieron en el material de RAEE triturado sin ningún tipo de recubrimiento; las composiciones de cada una se describen en la Tabla 1. 
Tabla 1-Composición de las distintas muestras realizadas ${ }^{\mathrm{a}}$.

\begin{tabular}{|c|c|c|c|c|c|}
\hline Entrada & Muestra & $\begin{array}{l}\text { Plástico de RAEE } \\
\text { (\% en peso) }\end{array}$ & $\begin{array}{l}\text { Cemento de } \\
\text { albañilería (\% en } \\
\text { peso) }\end{array}$ & $\begin{array}{l}\text { Carbón activado Tipo - } \\
\text { (\% en peso) }\end{array}$ & Tamaño $(\mathrm{mm})$ \\
\hline 1 & $\mathrm{~A}$ & 43 & 52 & CAE-061 - 5 & 3 \\
\hline 2 & $\mathrm{~B}$ & 44 & 53 & CAE-061 - 3 & 3 \\
\hline 3 & $\mathrm{C}$ & 43 & 52 & CAE-U - 5 & 8 \\
\hline 4 & $\mathrm{D}$ & 44 & 53 & CAE-U - 3 & 3 \\
\hline 5 & $E$ & 44 & 53 & CAE-U - 3 & 8 \\
\hline 6 & $\mathrm{~F}$ & 45 & 55 & --- & 3 \\
\hline 7 & $\mathrm{G}$ & 45 & 55 & --- & 8 \\
\hline 8 & RAEE 3 & 100 & --- & --- & 3 \\
\hline 9 & RAEE 8 & 100 & --- & --- & 8 \\
\hline
\end{tabular}

${ }^{a}$ La cobertura de todos los AS se realizó en tres capas.

Sobre estas muestras de material compuesto y RAEE molido se estudiaron sus propiedades físicas como granulometría y características morfológicas, propiedades mecánicas como resistencia a compresión y flexión y se realizó un análisis químico para determinar el efecto de los aditivos en los contaminantes orgánicos y metales pesados.

\section{Propiedades morfológicas}

Los descriptores de tamaño y forma fueron definidos mediante fotografías digitales de los distintos AS y su posterior análisis con el software Image J 1.49i. Según los resultados obtenidos, los descriptores de forma, como la redondez y la circularidad de las partículas de RAEE (3 y 8) aumentan al estar cubiertas con el encapado en todas las dosificaciones. Particularmente, la circularidad aumenta más cuando las partículas son de $3 \mathrm{~mm}$, como es el caso de A donde este parámetro pasa de 0,5 a 0,8. Por otro lado, la relación de aspecto disminuye si se comparan las partículas de RAEE (3 y $8 \mathrm{~mm}$ ) respecto a los AS. Este hecho indica que la carga del material del shell ocurre mayoritariamente en los lados más largos de las partículas, obteniendo así partículas con formas más esféricas que las que le dieron origen.

Con respecto a la densidad aparente, se observa una diferencia importante entre las muestras de RAEE y las encapadas con cemento, la densidad es poco menos que el doble. Para las muestras de RAEE 8 el valor de la densidad aparente pasa de $0,57 \mathrm{~g} / \mathrm{cm} 3$ a $0,83 \mathrm{~g} / \mathrm{cm} 3$ y $0,84 \mathrm{~g} / \mathrm{cm} 3$ para $\mathrm{C} \mathrm{y} \mathrm{G}$ respectivamente; el valor para el AS que posee CA es levemente menor que el que no posee CA. Algo similar se observa para las muestras de RAEE de $3 \mathrm{~mm}$, donde la densidad aparente aumenta desde $0,54 \mathrm{~g} / \mathrm{cm} 3$ a 0,80 g/cm3, 0,81 g/cm3, 0,78 g/cm3, 0,84 g/cm3 para A, B, D y F, respectivamente. Es importante destacar que, cuando el CA es el mismo pero las proporciones son diferentes la densidad aparente prácticamente no se ve afectada, disminuyendo muy poco cuanto más CA tiene el encapado. Sin embargo, cuando se comparan dos muestras con la misma proporción de CA, pero de diferente calidad (B vs. D) sí se observa un cambio en la densidad aparente del AS que pasa de $0,81 \mathrm{~g} / \mathrm{cm} 3$ a 0,78 g/cm3.

En comparación al agregado fino natural; que posee un valor de densidad aparente de 1,48 $\mathrm{g} / \mathrm{cm} \otimes[22]$, los AS poseen una densidad aparente menor, lo que implica a priori que la conductividad térmica de un mortero elaborado con AS será menor a la de un mortero elaborado con un agregado natural como la arena gruesa[16].

Los módulos de finura fueron determinados bajo la norma IRAM 1505 y resultaron mayores a los del agregado fino natural, esto se debe a que el tamaño de las partículas es mayor en todos los casos. Esto puede observarse en la Figura 1 donde se presentan las curvas granulométricas de las muestras y el agregado fino natural. Se observa que las muestras que más se asemejan a la curva límite indicada por el Reglamento CIRSOC 201-05 y norma IRAM 1627, y con la cual el agregado fino natural coincide correctamente; son A, F, D y RAEE-3, las cuales presentan valores más 
cercanos de módulo de finura de dicho agregado. Otra particularidad que presentan estas muestras es que todas están hechas con un tamaño de partícula de plástico de $3 \mathrm{~mm}$.

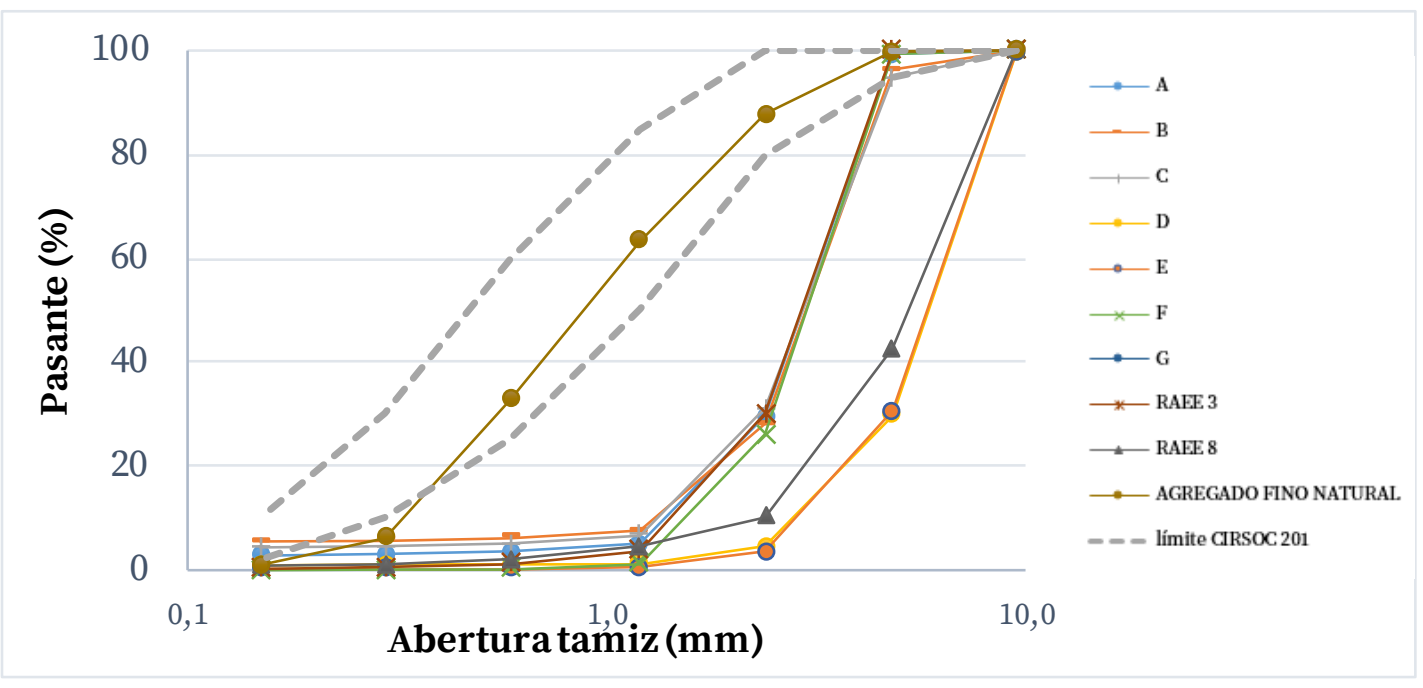

Figura 1 -Distribución de tamaños de partícula por tamices según norma IRAM 1505.

Comportamiento mecánico

Los AS pueden ser utilizados como reemplazo del agregado fino natural en morteros cementicios. Su desempeño en la resistencia a la compresión simple y flexión fue determinado ensayando probetas normalizadas de acuerdo a la Norma IRAM 1622 a 28 días de fraguado, arrojando valores inferiores al agregado natural pero aceptables para morteros. Se fabricaron probetas de partículas de FP-RAEE y AS en granulometrías 3 y $8 \mathrm{~mm}$ con cemento como aglutinante. Todas las probetas se elaboraron con una relación en volumen de agregado: cemento 6:1 y una relación en peso de agua: cemento de 0,5 .

Los valores de resistencia a la compresión resultaron aceptables para componentes constructivos, mientras que las probetas que resultaron aptas para ser empleadas como elemento constructivo portante según la norma IRAM 11561 arrojaron un valor promedio de 4,46 MPa. Esta probeta fue fabricada con AS encapado sólo con cemento, sin carbón activado (F); existen artículos publicados que demuestran que el carbón activado retarda el fraguado del cemento Portland [17], lo cual provoca una leve disminución en las propiedades mecánicas finales de la pieza. Otra particularidad a tener en cuenta es el tamaño del AS con que está fabricada la probeta (en este caso es $3 \mathrm{~mm}$, que posee una curva granulométrica similar a la del agregado fino natural), esto se ve reflejado también en su resistencia mecánica debido a la mayor compactación/distribución entre las partículas. Para el caso de la resistencia obtenida por la probeta $\mathrm{G}$, se produce una disminución significativa en la resistencia respecto a $\mathrm{F}$, lo que hace suponer que el tamaño del AS con que está formado el mortero de la probeta, condiciona su resistencia mecánica final. Esto también afecta a la curva granulométrica que aparece alejada de la del agregado natural; la probeta está formada con las mismas cargas y dosificación que $\mathrm{F}$, pero es el tamaño de partícula el cual genera esta significativa diferencia entre las curvas. Si bien la resistencia a la compresión del agregado natural es de 6,3 MPa, el valor de resistencia mecánica de F es suficiente para cumplir como mampuesto estructural. Esta misma probeta arrojó también el mayor valor de resistencia la flexión; 2,0 $\mathrm{MPa}$, lo que la convierte en la mejor alternativa en cuanto a resistencia mecánica. La probeta con CA que mejor resistencia a la compresión y flexión obtuvo fue A, que resiste 3,3 $\pm 0,3 \mathrm{MPa}$ para

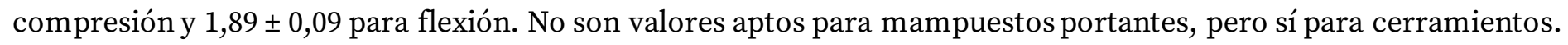

\section{Análisis químico}

En relación a la neutralización de metales pesados, el cemento resultó eficiente para neutralizar el antimonio (Sb). Se sometió a las partículas de RAEE a una digestión acida en agua regia a 80 grados. Los valores de $\mathrm{Sb}$ extraídos 
resultaron muy cercanos al 100\% de la composición; $741 \pm 10 \mathrm{mg} / \mathrm{Kg}$. Cuando se realizó una extracción en agua destilada a 100 grados, los valores disminuyeron significativamente; a 23,6 6 0,5 mg/Kg. Se realizó el mismo ensayo para la partícula de A.S, el resultado fue aún menor: $6 \pm 0,3 \mathrm{mg} / \mathrm{Kg}$. Lo mismo sucedió con el Zinc (Zn), que pasó de $155 \pm 4$ estando la partícula sin recubrir a $0.188 \pm 0.003$ con recubrimiento. De todas maneras, para el caso del Zn, se deprende un valor muy similar en el ensayo con el agua destilada a 100 grados, la partícula sin recubrir y la recubierta arrojaron un valor muy similar ( $0.176 \pm 0.002$ y $0.188 \pm 0.003$ respectivamente) esto se debió a que, al estar sometido el árido a altas tem peraturas se rompe, dejando caras de plástico en contacto directo con el agua, arrojando entonces los valores correspondientes al plástico y no al recubrimiento con cemento.

Otro metal que se neutralizó eficientemente fue el cobre $(\mathrm{Cu})$, quedando bajo el límite cuantificación mediante el procedimiento de lixiviación característica de toxicidad (TCLP).

Para el caso de los contaminantes orgánicos, se analizó el lixiviado de TBBPA en agua para partículas de RAEE y para AS con CA y sin CA mediante extracciones en agua a 50 y 100 grados. También se analizó el agua de curado de las mismas. Para el caso de las lixiviaciones con agua a 100 grados fue significativa la diferencia entre el árido que contiene carbón activado (A) y el que no (F). F al no contener carbón activado lixivia casi 2000 veces más ppm que A. Incluso 2000 veces más que la partícula de plástico sin recubrir. Esto se debe a la naturaleza intrínseca del TBBPA que tiene dos grupos hidroxilo aromáticos ionizables (grupos funcionales de fenol) [18] dándole débiles propiedades ácidas. Cuando el TBBPA toma contacto con el cemento, que tiene un comportamiento básico conocido [19], reacciona inmediatamente para formar las especies aniónicas del TBBPA.

Para los ensayos con agua a 100 grados y con aguas de curado, sucede el mismo efecto, pero las cantidades lixiviadas para A resultaron bajo los límites de cuantificación y detección.

Se tiene como conclusión entonces que el árido que neutraliza eficazmente tanto metales como componentes orgánicos es A, porque presenta las capas de cemento que neutralizan metales y capas de CA que neutralizan contaminantes orgánicos mientras que controlan la lixiviación que el cemento provoca. Siempre y cuando no se someta al árido a temperaturas mayores a 100 grados. A su vez, es el árido que, después de F, presenta mejores propiedades mecánicas para mampuestos constructivos de cerramiento.

\section{Ensayos que se están desarrollando en la actualidad}

Conductividad térmica de los mampuestos de AS

Análisis de Ciclo de vida

Resistencia al ataque de ácidos y álcalis

\section{Ensayos próximos a desarrollar}

Permeabilidad al vapor de agua

Análisis de costos

Desarrollo de placas, vigas, bloques pre moldeados, etc

\section{Referencias}

[1] N. Singh, D. Hui, R. Singh, I. P. S. Ahuja, L. Feo, and F. Fraternali, "Recycling of plastic solid waste: A state of art review and future applications,” Compos. Part B Eng., vol. 115, pp. 409-422, 2017.

[2] Global Waste Management Outlook. 2016.

[3] W. Tamaddon, F., \& Hogland, "Review of Cadmium in Plastic Waste in Sweden," Waste Manag. Res. 11(4), 287295., 1993.

[4] E. Dimitrakakis, A. Janz, B. Bilitewski, and E. Gidarakos, "Determination of heavy metals and halogens in plastics from electric and electronic waste," Waste Manag., vol. 29, no. 10, pp. 2700-2706, 2009.

[5] E. Stenvall, S. Tostar, A. Boldizar, M. R. S. J. Foreman, and K. Möller, "An analysis of the composition and metal contamination of plastics from waste electrical and electronic equipment (WEEE)," Waste Manag., vol. 33, no. 4, pp. 915-922, 2013.

[6] Greenpeace, "El lado toxico de la telefonía movil," 2011.

[7] L. Devia, “Basura electrónica: los riesgos de consumir y descartar,” Saber Cómo, vol. no. 108, p. 2, 2012.

[8] E. Maris, P. Botané, P. Wavrer, and D. Froelich, "Characterizing plastics originating from WEEE: A case study in France," Miner. Eng., vol. 76, pp. 28-37, 2015. 
[9] S. A. Unnisa and M. Hassanpour, "Development circumstances of four recycling industries (used motor oil, acidic sludge, plastic wastes and blown bitumen) in the world," Renew. Sustain. Energy Rev., vol. 72, no. January, pp. 605-624, 2017.

[10] CARPL, "Estudio sobre los retardantes de llama bromados en los países del Mediterráneo," Cent. Act. Reg. para la Prod. Limpia, 2010.

[11] A. Aldrian, A. Ledersteger, and R. Pomberger, "Monitoring of WEEE plastics in regards to brominated flame retardants using handheld XRF," Waste Manag., vol. 36, pp. 297-304, 2015.

[12] A. Report and S. F. T. R. Ta-, "Final Report of Phase I of the ACAP Project on Brominated Flame Retardants (BFRs)," 2008.

[13] A. Haarman and M. Gasser, Managing hazardous additives in WEEE plastic from the Indian informal sector, no. June. 2016.

[14] L. Gu and T. Ozbakkaloglu, "Use of recycled plastics in concrete: A critical review," Waste Manag., vol. 51, pp. 1942, 2016.

[15] W. Schlummer, M., Mäurer, A., Leitner, T., \& Spruzina, "Report: Recycling of flame-retarded plastics from waste electric and electronic equipment (WEEE).," Waste Manag. Res. 11(4), 287-295., vol. 24(6), pp. 573-583, 2006.

[16] J. L. Ruiz-Herrero et al., "Mechanical and thermal performance of concrete and mortar cellular materials containing plastic waste," Constr. Build. Mater., vol. 104, pp. 298-310, 2016.

[17] “Master de Ciencia y Tecnología de Materiales Empleo de adsorbentes como aditivos en cementos :," 2014.

[18] A. Bergman et al., "A novel abbreviation standard for organobromine, organochlorine and organophosphorus flame retardants and some characteristics of the chemicals," Environ. Int., vol. 49, pp. 57-82, 2012.

[19] B. J. y K. A. Birchall James Derek, Howard AJ, "Sobre la hidratación del cemento Portland," Proc. R. Soc. Lond. A, vol. 360 . 